\title{
Metástasis intramedular como primera manifestación de un carcinoma renal
}

\author{
A. Gómez de la Riva; A. Isla; C. Pérez-López; M. Budke; M. Gutiérrez y R Frutos
}

Servicio de Neurocirugía. Hospital Universitario La Paz. Madrid.

\section{Resumen}

Presentamos el caso de un varón de 69 años sin patología previa de interés que presentó clínica de pérdida de fuerza distal en miembros inferiores de dos meses de evolución. La resonancia magnética (RM) mostró la presencia de una masa intramedular a nivel del cono. El paciente fue intervenido practicándose resección de la tumoración, mostrando el estudio histológico la presencia de un carcinoma de células claras correspondiente a una sospecha de metástasis de un tumor renal, que posteriormente se confirmó con una tomografía axial computarizada (TAC) abdominal. Cuando el estado del paciente es bueno, la cirugía puede subsanar el déficit neurológico producido por la lesión intramedular. Nuestro paciente, tras la intervención, mejoró su estado neurológico; y actualmente, tras 14 meses, se mantiene sin déficit neurológico en miembros inferiores.

PALABRAS CLAVE: Metástasis. Intramedular. Carcinoma renal.

Intramedullary spinal cord metastasis as the first manifestation of a renal carcinoma

\section{Summary}

We present the case of a 69 year-old male without known antecedente who presented a clinical loss of distal force in his lower limbs during the last two months. Magnetic resonance imaging (MRI) showed an intramedullary spinal cord mass at the level of the medullaris cone. The patient was operated on; the histological diagnosis was clear cells carcinoma suggestive of metastasis from an unsuspected renal tumor that was later confirmed with an abdominal CT. When the patient's state is good, surgery can correct the neurological deficit produced by an intramedullary spinal cord lesion. The neurological state of our patient impro-

Recibido: 18-08-04. Aceptado: 29-11-04 ved after the intervention, and $\mathbf{1 4}$ months after surgery, he has no neurological deficit in the lower limbs.

KEY WORDS: Metastasis. Intramedullary spinal cord. Renal carcinoma.

\section{Introducción}

Las metástasis intramedulares ocurren, dependiendo de las series, entre un 0.85 y un $3.9 \%$ de los tumores metastáticos que afectan a la columna espinal ${ }^{24}$. Además, sólo se encuentran en un $2 \%$ de los pacientes que fallecen por metástasis sistémicas ${ }^{3}$. De cualquier modo, con el avance de los métodos diagnósticos (RM) y los protocolos quimioterápicos, se está produciendo un aumento del número de metástasis intramedulares diagnosticadas ${ }^{8,13}$. El diagnóstico debe ser confirmado con una biopsia de la lesión, además de otros signos de su cáncer primitivo cuando están presentes ${ }^{3}$. Presentamos un caso clínico de una metástasis en el cono medular procedente de un carcinoma renal de células claras y cuya primera manifestación fue un déficit motor de miembros inferiores.

\section{Caso clínico}

Varón de 69 años que consulta en urgencias refiriendo un cuadro de disminución de fuerza en miembro inferior derecho de unos quince días de evolución, progresivo y sin causa aparente. Presentaba, además, parestesias en zonas distales de ambos pies. No refería lumbalgia y el cuadro se inició tras un dolor en la rodilla. No existían antecedentes de interés a excepción de hipertensión arterial tratada y bien controlada. A la exploración por el médico de urgencias se observó marcha en "stepage" y una disminución de fuerza 4/5 para la flexión dorsal del pie derecho. Fue diagnosticado en urgencias de posible neuropraxia del ciático poplíteo externo derecho y se le remitió a consulta de rehabilitación, donde, dos meses después del comienzo de la clínica se le remite al servicio de neurocirugía tras observar en la RM una lesión intramedular a nivel de L1, redondeada, mal definida, de $1 \times 1 \mathrm{~cm}$., con centro isointenso y 


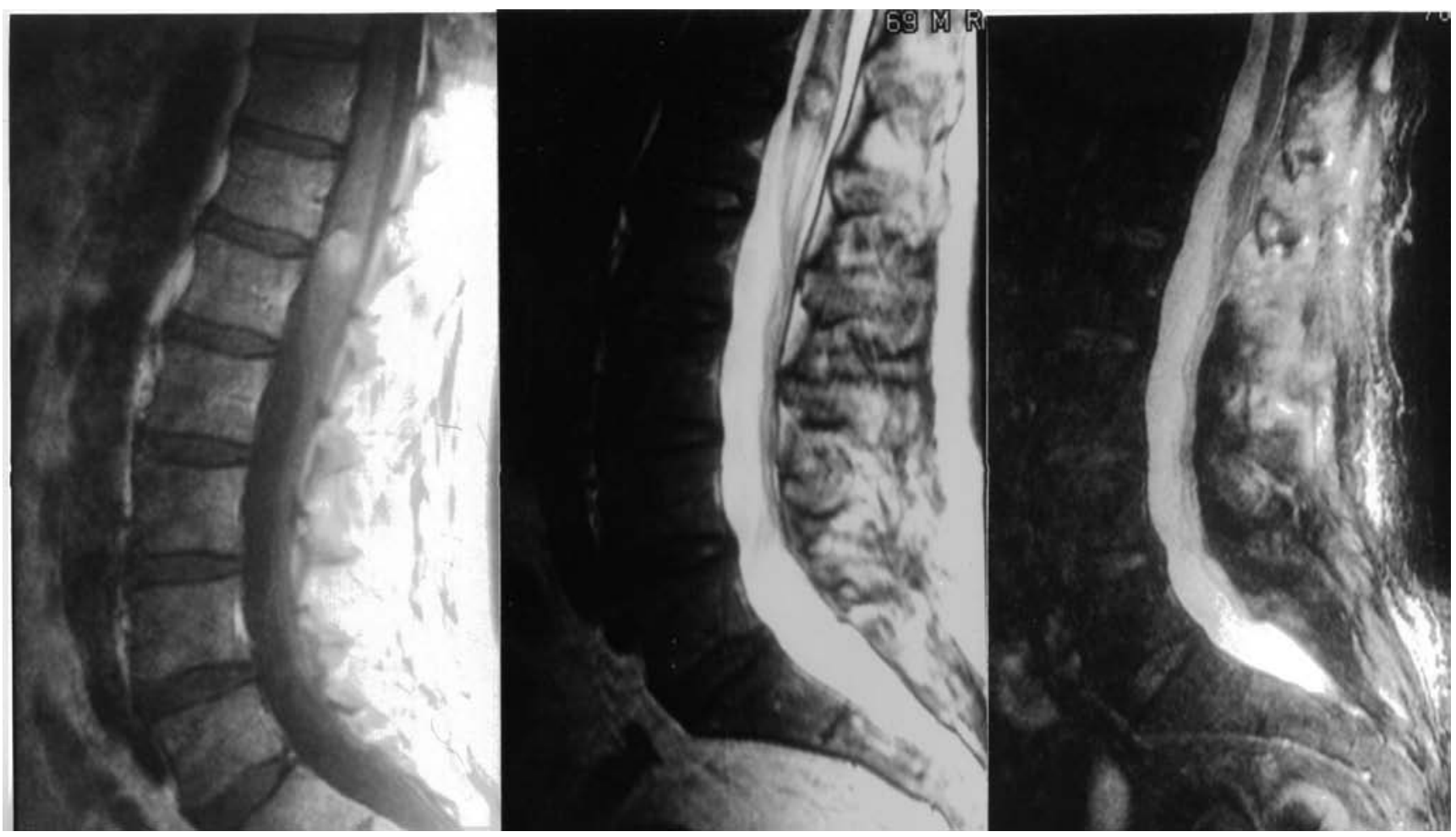

Figura 1. Izquierda: RM sagital potenciada en T1 con contraste muestra la presencia de una masa redondeada a nivel de cono medular. Centro: Imagen en RM sagital potenciada en T2 mostrando tumoración intramedular bien definida con edema perilesional. Derecha: RM potenciada en T2 realizada 3 meses tras la intervención quirúrgica no muestra restos de tumoración ni edema.

periferia hipointensa en $\mathrm{T} 1$, que tras la administración de gadolinio realza de forma difusa y que muestra engrosamiento del diámetro del cono medular (Fig. 1 izquierda y centro). Como primer diagnóstico se informó la posibilidad de un ependimoma de cono medular sin poder descartar que se tratase de un astrocitoma. No se hizo mención a ninguna masa a excepción de la anteriormente descrita y en la RM no se evidenciaba otro proceso. El paciente fue ingresado en el servicio de neurocirugía presentando hipoestesia en puntas de dedos de ambos pies, junto disminución de fuerza distal de $2 / 5$ en miembro inferior derecho y de $3 / 5$ en miembro inferior izquierdo. Conservaba a su ingreso el control de los esfínteres y el índice de Karnofsky era de 70. La radiología de tórax fue informada como normal.

Se intervino al paciente practicándose una laminotomía osteoplástica y una resección total de una masa de 1 x 1 $\mathrm{cm}$., fácilmente resecable y poco hemorrágica. El estudio histológico demostró la presencia de una metástasis de carcinoma de células claras de probable origen renal (Fig. 2). Este diagnóstico se confirmó con la realización de una TAC tóraco-abdominal, en el que se observó una masa gigante renal $(11 \times 10 \times 14 \mathrm{~cm}$.) (Fig. 3) y diversas metástasis abdominales y pulmonares de tamaño menor a $1 \mathrm{~cm}$. no evidenciables con la radiología simple. El paciente recu- peró prácticamente en su totalidad la paraparesia durante su estancia en el servicio de neurocirugía. El estudio con RM de control realizado a los 3 meses no mostró ningún signo de recidiva (Fig. 1 derecha). El paciente evolucionó favorablemente, sin presentar sintomatología neurológica, habiéndose cumplido 14 meses desde su intervención.

\section{Discusión}

Las metástasis intramedulares son infrecuentes, representando alrededor del $2 \%$ de todos los tumores intramedulares, lo cual puede ser resultado del pequeño tamaño de la médula espinal y de lo apartado que se encuentra de la accesibilidad vascular para la embolización tumoral ${ }^{3,10}$. Estas metástasis, clínicamente, afectan sólo al $0.1-0.4 \%$ de todos los pacientes con cáncer metastático. Además, es poco frecuente que la enfermedad metastática comience con la afectación intramedular como primer síntoma, como ocurrió con nuestro paciente.

El origen más frecuente de las metástasis intramedulares corresponde al cáncer microcítico de pulmón $(54 \%)^{2,13}$, que típicamente metastatiza vía hematógena ${ }^{30}$, seguido del cáncer de mama (13\%), y en menor frecuencia el melanoma maligno, linfoma y cáncer de colon ${ }^{10,17}$. El carcinoma renal 


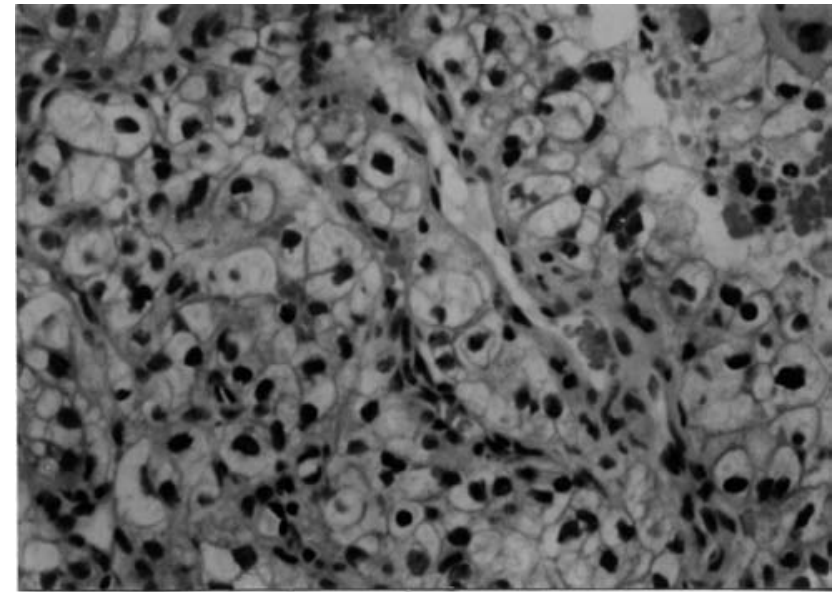

Figura 2. Células tumorales con citoplasma claro (HEX 400).

se disemina preferentemente en pulmones (50\%), huesos (49\%), ganglios linfáticos $(6-32 \%)$, hígado $(8 \%)$ y cerebro $(3 \%)^{23}$. Este carcinoma no tiene como lugar típico de diseminación la médula espinal, aunque es dado a metastatizar en lugares poco frecuentes. La metástasis intramedular se suele producir por vía arterial, estando respetados los tejidos leptomeníngeos, como es el caso de nuestro paciente. También se puede producir por vía venosa a través del plexo de Batson o a través de diseminación directa en raíces dorsales ${ }^{22}$.

En el carcinoma renal, las metástasis se presentan en un $37 \%$ de los casos, siendo más frecuente la enfermedad metastásica múltiple que la única ${ }^{6}$.

En cuanto a la clínica, el tiempo de evolución de los síntomas en los tumores intramedulares metastáticos es de semanas o pocos meses, mientras que los tumores primarios suelen evolucionar en un rango de 2-3 años ${ }^{2}$. La clínica de estos pacientes viene dominada por el dolor, siendo la presentación más frecuente el dolor local, que aumenta con las maniobras de Valsalva y que hace despertar al paciente por la noche. Las alteraciones motoras y sensoriales también son frecuentes y se relacionan con el nivel afectado. Cuando ocurren en el cono medular es frecuente la aparición de alteraciones de esfínteres, sobre todo en el control de la orina, e impotencia. En nuestro paciente el diagnóstico inicial fue difícil, debido a que sólo presentó al inicio del cuadro una leve disminución de fuerza para la flexión dorsal del miembro inferior derecho. Este caso de primera manifestación de un carcinoma renal es extremadamente raro, habiéndose publicado sólo otro caso en la literatura ${ }^{27}$. En el diagnóstico de las lesiones intramedulares es necesario la realización de una RM, debido a que es la técnica más sensible para el diagnóstico de lesiones en esta localización, además, es útil la administración de gadolinio para producir un realce de la lesión. La RM también permite

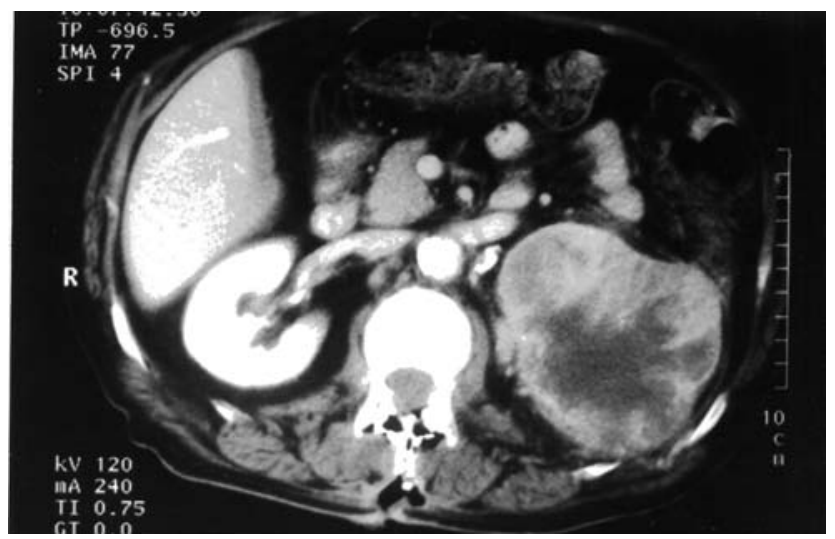

Figura 3. TAC abdominal con contraste realizada a los 10 días de la intervención quirúrgica que muestra la presencia de gran tumoración renal izquierda.

el posible diagnóstico de otras metástasis espinales o cerebrales. Las imágenes potenciadas en T2 proporcionan una ayuda adicional al mostrar áreas de edema asociadas ${ }^{2}$, mientras que una señal normal puede excluir la metástasis intramedular ${ }^{18}$.

La histología de las lesiones intramedulares lleva al diagnóstico de la enfermedad y permite la diferenciación de las lesiones primarias de los raros casos de metástasis intramedular. Sin embargo, puede resultar difícil distinguir entre metástasis por cáncer renal de células claras y hemangioblastoma primario (como en el caso de nuestro paciente), cuya frecuencia no es despreciable (3-8\% de los tumores intramedulares $)^{28}$, debido a las similitudes que presentan en el estudio convencional, por lo que es necesario realizar técnicas inmunohistoquímicas para diferenciarlos. El carcinoma renal de células claras presenta positividad para el antígeno epitelial de membrana (EMA) y para queratinas, siendo negativo para la proteína ácido gliofibrilar (PAGF), mientras que en el hemangioblastoma ocurre lo contrario $^{1}$. En un $25 \%$ de los casos de hemangioblastoma se puede diferenciar debido a que van acompañados del síndrome de Von Hippel-Lindau ${ }^{20}$.

El estudio histológico tiene valor pronóstico, debido a que los subtipos de tumores renales con células claras o células granulares tienen mejor pronóstico que los subtipos con células en huso o anaplásicas ${ }^{7,26}$. De cualquier modo, algunos estudios revelan que no hay dicha diferencia ${ }^{14}$.

En cuanto al tratamiento, algunos cirujanos abogan por la cirugía radical para obtener el diagnóstico histológico de la tumoración, si no se conocía previamente el origen, y para tratar de mejorar el estado neurológico del paciente, puesto que la supervivencia media de estos pacientes es de 4-7 meses, dependiendo del cáncer primario. En determinados casos, como los tumores primarios o las metástasis que previamente no estaban diagnosticadas, como es el caso de nuestro paciente, la resección total de la lesión 
puede mejorar su estado de déficit neurológico, su calidad de vida y la supervivencia ${ }^{4,12,16,29,32}$. Si el paciente estaba diagnosticado previamente del tumor primario habría que tener en cuenta el índice de Karnofsky, junto con el pronóstico del tumor primitivo para decidir la intervención quirúrgica. Actualmente, en los pacientes con procesos tumorales intraespinales realizamos de forma sistemática una osteotomía osteoplástica con microplacas, cuya técnica describió Wiedemayer y cols. ${ }^{31}$, por dos motivos principalmente: el primero es evitar la fibrosis que se produce después de la laminectomía dificultando la posibilidad de una reintervención cuando se produce una recidiva, estando indicada esta técnica en nuestro paciente debido a la sospecha de que padecía un tumor primario. El segundo motivo es que con la osteotomía osteoplástica se consigue restaurar el canal (reestableciendo la tercera columna de Denis ${ }^{5}$ ) consiguiéndose una mayor estabilidad de la columna intervenida sobre todo cuando hay que realizar laminectomía de dos o más niveles.

En un $62-85 \%$ de los pacientes con metástasis intramedulares se encuentran también metástasis intracerebrales ${ }^{32}$, por lo que está indicada la realización de una RM o TAC cerebral, que en nuestro paciente fue normal.

Debido a la enfermedad de base de nuestro paciente (carcinoma renal de células claras) es aconsejable el tratamiento adyuvante, ya sea radioterapia, quimioterapia con 5-fluoracilo, o inmunoterapia con interferón- $\alpha-2 b$ o interleuquina 2 (IL2) ${ }^{19,25}$. En nuestro caso, el paciente y su familia rechazaron todo tipo de tratamiento enfocado al tumor primitivo y a las metástasis en otras localizaciones, tanto abdominales como torácicas.

Diversos estudios sugieren la significativa diferencia, en términos de supervivencia, entre pacientes con metástasis únicas y pacientes con metástasis múltiples ${ }^{6,21}$. La supervivencia media de la enfermedad metastásica en el carcinoma renal es de 17 meses (rango 2-207 meses) para pacientes con metástasis única, y 6 meses (rango 1-132 meses) para pacientes con enfermedad metastásica múltiple ${ }^{6}$. En pacientes con metástasis intramedulares, la supervivencia media, tras el comienzo de los síntomas neurológicos, es de 3 meses en el estudio de Grem y cols. ${ }^{15}$; de 2 semanas a 9 meses en el estudio de Dunne y cols ${ }^{9}$; de 13 meses en el caso de Schijns y $\operatorname{cols}^{27}$; o de 4 a 23 meses en la serie de Fakir y cols ${ }^{11}$. Nuestro paciente continua con vida y sin síntomas neurológicos tras 14 meses de la cirugía.

\section{Conclusiones}

La cirugía radical de las lesiones intramedulares, ya sea curativa o paliativa, puede ser beneficiosa en pacientes en los que los síntomas neurológicos avanzan rápidamente. Es importante el estado general del paciente para decidir la cirugía y una correcta evolución postoperatoria.

\section{Bibliografía}

1. Banner, B.F., Burnham, J.A., Bahnson, R.R., Ernstoff, M.S., Auerbach, H.E.: lmmunophenotypic markers in renal cell carcinoma. Mod Pathol. 1990, 3: 129-134.

2. Connolly, E.S., Winfree, C.J., McCormick, P.C., Cruz, M., Stein, B.M.: Intramedullary spinal cord metastasis: Report of three cases and review of the literature. Surg Neurol 1996; 46: 329-338.

3. Costigan, D.A., Winkelman, M.D.: Intramedullary spinal cord metastasis: A clinicopathological study of 13 cases. J Neurosurg 1985; 62: 227-233.

4. Decker, R.E., Sundrani, S, Citron, M.L., Herrschaft, D.S.: Intramedullary spinal cord metastasis treated by complete resection of tumor prior to radiotherapy and chemotherapy. Spine 1987; 12: 393-395.

5. Denis, F.: The three column spine and its significance in the classification of acute thoracolumbar spinal injuries. Spine 1983; 8: 817-831.

6. Dineen, M.K., Pastore, R.D., Emrich, L.J., Huben, R.P.: Results of surgical treatment of renal cell carcinoma with solitary metastasis. J Urol 1988; 140: 277-279

7. Dinney, C.P., Awad, S.A., Gajewski, J.B., et al: Analysis of imaging modalities, staging systems, and prognosis indicators of renal cell carcinoma. Urology. 1992; 39 : 122-129.

8. Dune, J.W., Harper, C.G., Pamphlett, R.: Intramedullary spinal cord metastasis. Neurology 1979; 22: 1222-1231.

9. Dunne, J.W., Harper, C.G., Pamphlett, R.: Intramedullary spinal cord metastases: a clinical and pathological study of nine cases. Q J Med 1986; 61: 1003-1020.

10. Edelson, R.N., Deck, M.D.F. and Posner, J.B.: Intramedullary spinal cord metastasis. Neurology 1979; 22: 12221231.

11. Fakih, M., Schiff, D., Erlich, R., Logan, TF.: Intramedullary spinal cord metastasis (ISCM) in renal cell carcinoma: a series of six cases. Ann Oncol. 2001; 12: 1173-1177.

12. Findlay, J.M., Bernstein, M., Vanderlinden, R.G., Resch, L.: Microsurgical resection of solitary intramedullary spinal cord metastases. Neurosurgery 1987; 21: 911-915.

13. Fornari, M., Puchino, F., Solero, C.L., et al.: Microsurgical treatment of intramedullary spinal cord tumors. Acta Neurochir Suppl (Wien) 1988; 43: 3-8.

14. Golimbu, M., Al-Askari, S., Tessler, A., Morales, P.: Aggresive treatment of metastatic renal cell cancer. J Urol 1986; 136: 805-807.

15. Grem, J.L., Burgess, J., Trump, DL.: Clinical features and natural history of intramedullary spinal cord metastasis. Cancer 1985; 56: 2305-1214.

16. Isla, A., Paz, J.M., Sansivirini, F., Zamora, P., Garcia Grande, A., Fernández, A.: Intramedullary spinal cord metastasis. A case report. J Neurosurg Sci 2000, 44: 99-101.

17. Jellinger, K., Kothbaue,r P., Sunder-Plassmann, et al.: 
Intramedullary spinal cord metastases. J Neurol 1979; 220: 31-41.

18. Kamholtz, R., Sze, G.: Current imaging in spinal metastatic disease. Semin Oncol 1991; 18: 158-169.

19. Leibovich, B.C., Han, K.R., Bui, M.H., et al: Scoring algorithm to predict survival after nephrectomy and immunotherapy in patients with metastatic renal cell carcinoma: a stratification tool for prospective clinical trials. Cancer 2003; 98: 2566-2575.

20. Neumann, H.P.H., Eggert, H. R., Weigel, K., et al.: Hemangioblastomas of the central nervous system: A 10-year study with special reference to von hippellindau syndrome. J Neurosurg 1989; 70: 24-30.

21. Neves, R.J., Zincke, H., Taylor, W.F.: Metastatic renal cell Cancer and radical nephrectomy: identification of prognostic factors and patients survival. J Urol 1988; 139: 11731176.

22. Olson, M.E., Chernick, N.L., Posner, J.B.: Infiltration of the leptomeninges by systemic Cancer. Arch Neurol 1974; 30: 122-137.

23. Pagano, S., Frazoso, F., Ruggeri, P.: Renal cell carcinoma metastasis. Scand J Urol Nephrol 1996; 30: 165-172.

24. Posner, J.B.: Neurological complications of systemic Cancer. Med Clin North Am 1971; 55: 625-646.

25. Ravaud, A., Trufflandier, N., Ferriere, J.M., et al.: Subcutaneous interleukin-2, interferon alpha-2b and 5-fluorouracil in metastatic renal cell carcinoma as second-line treatment after failure of previous immunotherapy: a phase II trial. Br J Cancer 2003; 89: 2213-2218.

26. Reis, M., Faria, V.: Renal cell carcinoma. Reevaluation of prognostic factors. Cancer 1988; 61: 1192-1199.

\section{Comentario al trabajo: Metástasis intramedular como primera manifestación de un carcinoma renal de Gómez de la Riva y cols.}

Los autores presentan el caso de un paciente sin antecedentes oncológicos conocidos, que debuta con una paraparesia secundaria a una metástasis intramedular, como primera manifestación de un carcinoma renal.

Se trata de un caso excepcional por varias razones. En primer lugar, la incidencia de las metástasis intramedulares es muy baja, representando en torno al $2 \%$ de los tumores intramedulares, ya poco frecuentes, de por si, en la práctica neuroquirúrgica. En segundo lugar por su origen, ya que el adenocarcinoma renal representa sólo un 9\% de las metástasis intramedulares. El origen pulmonar es el más frecuente $(54 \%)$ seguido del cáncer de mama (11\%). El origen renal es el tercero en frecuencia según una reciente revisión
2005; 16: 359-364

27. Schijns, O.E., Kurt, E., Wessels, P., Luijckx, G.J., Beuls, E.A.: Intramedullary spinal cord metastasis as a first manifestation of a renal cell carcinoma: report of a case and review of the literature. Clin Neurol Neurosurg 2000; 102: 249-254.

28. Solomon, R.A., Stein, B.M.: Unusual spinal cord enlargement related to intramedullary hemangioblastomas. J Neurosurg 1988; 68: 550-553,.

29. Stranjanis, G., Torres, M.J.: Successful removal of intramedullary spinal cord metastasis: a case report. Br J Neurosurg 1993; 7: 193-215.

30. Tomlinson, B.E., Perry, R.H., Stewart-Wynne, E.G.: Influence of the site of origin of lung carcinomas of clinical presentation and the central nervous systems metastases. J Neurol Neurosurg Psychiatry 1979; 42: 82-88.

31. Wiedemayer, H., Schoch, B., Stolke, D.: Osteoplastic laminotomy using titanium microplates for reconstruction of the laminar roof. a technical note. Neurosurg Rev. 1998; 21: 93-97.

32. Winkleman, M.D., Adelstein, D.J., Karlins, N.L.: Intramedullary spinal cord metastasis: diagnosis and therapeutic considerations. Arch Neurol 1987; 44: 526-531.

Gómez de la Riva, A.; Isla, A.; Pérez-López, C.; Budke, M.; Gutiérrez, m.; Frutos, R.: Metástasis intramedular como primera manifestación de un carcinoma renal. Neurocirugía 2005; 16: 359-364.

Correspondencia postal: A. Gómez de la Riva. Servicio de Neurocirugía. Hospital Universitario La Paz. Paseo de la Castellana, 261. 28046 Madrid

de Kalacyi et al $^{1}$, algo más que lo que aportan las revisiones clásicas, seguido del melanoma (8\%) y el linfoma (4\%). En tercer lugar, las metástasis intramedulares suelen aparecer en pacientes con enfermedad neoplásica conocida, aunque en una cuarta parte de los casos puede ser el debut de la enfermedad $^{1}$. En el caso de las metástasis intramedulares de origen renal sólo hay un caso previo publicado ${ }^{2}$ en el que la metástasis se diagnosticó antes que el tumor primario. En cuarto lugar, el tratamiento quirúrgico sólo se realiza en menos de un tercio de los pacientes según las series más recientes. No existen guías de práctica clínica en cuanto al mejor tratamiento de estas metástasis. El tratamiento clásico ha sido la radioterapia acompañada 
de esteroides y/o quimioterapia según el tumor primario. En la revisión de Kalacyi et al ${ }^{1}$, se revisan los 138 casos publicados en la literatura de metástasis intramedulares desde 1987, realizándose exéresis quirúrgica sólo en 32 casos. La edad, situación basal y neurológica del paciente y el tipo y estadío tumoral son factores determinantes a la hora de sentar la indicación quirúrgica. También la ausencia de enfermedad neoplásica conocida es un factor a favor del tratamiento quirúrgico, ya que permite además el diagnóstico histológico del tumor intramedular. Parece que el tratamiento quirúrgico aporta una mejor recuperación funcional y una mayor supervivencia que el tratamiento radioterápico ${ }^{1}$. Para tumores radiorresistentes como el adenocarcinoma renal, la cirugía es el único tratamiento algo efectivo. En quinto lugar, la supervivencia media de los pacientes con metástasis intramedulares es de unos 7 meses ( 5 los no operados frente a 9.5 para los operados $)^{1}$ por lo que llama la atención que el paciente de nuestro caso siga vivo a los 14 meses sin haber recibido tratamiento para el tumor primario ni para las metástasis, aunque existen
2005; 16: A-B

casos publicados de supervivencias de hasta 2 años tras tratamiento quirúrgico de una metástasis intradural de un adenocarcinoma renal ${ }^{3}$.

\section{Bibliografía}

1. Kalacyi,, M., Çagavi F., Gül S., Yenidünya S., Açikgöz B.: Microsurgical resection of Intramedullary spinal cord metastases of large cell lung cancer. Acta Neurochir (Wien) 2004; published online September 2004.

2. Schijns, O.E., Kurt, E., Wessels, P., Luijekx, G.J., Beuls, E.A.: Intramedullary spinal cord metastasis as a first manifestation of a renal cell carcinoma: report of a case and review of the literature. Clin Neurol Neurosurg 2000; 102: 249-254.

3. Gaetani, P., Di leva, A., Colombo, P., Tancioni, F., Aimar, E., Debemardi, A., Rodriguez y Baena, R.: Intradural spinal metastasis of renal cell carcinoma causing cauda equina syndrome. Acta Neurochir (Wien) 2004; 146: 857-861.

A. de la Lama

Vigo 